\title{
Fuga de talento en la investigación española sobre Comunicación: percepción de los investigadores españoles en el extranjero
}

\section{The brain drain in Spanish Communication research: the perspective of Spanish academics abroad}

\author{
Francisco Seoane-Pérez; Manuel Martínez-Nicolás; Miguel Vicente-Mariño
}

Cómo citar este artículo:

Seoane-Pérez, Francisco; Martínez-Nicolás, Manuel; Vicente-Mariño, Miguel (2020). "Fuga de talento en la investigación española sobre Comunicación: percepción de los investigadores españoles en el extranjero". Profesional de la información, v. 29, n. 4, e290433.

https://doi.org/10.3145/epi.2020.jul.33

Artículo recibido el 26-01-2020

Aceptación definitiva: 21-04-2020

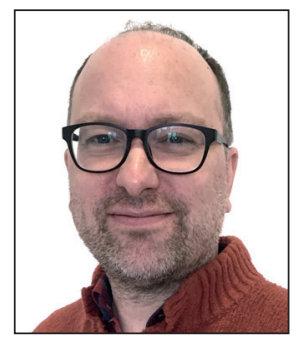

Francisco Seoane-Pérez

https://orcid.org/0000-0002-4680-558X

Universidad Carlos III de Madrid

Departamento de Comunicación

Madrid, 133

28903 Getafe (Madrid), España

francisco.seoane@uc3m.es

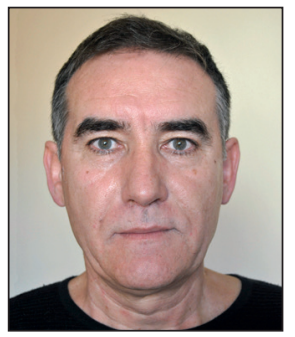

Manuel Martínez-Nicolás

https://orcid.org/0000-0002-3949-2351

Universidad Rey Juan Carlos

Facultad de Ciencias de la Comunicación

Camino del Molino, 5

28942 Fuenlabrada (Madrid), España

manuel.martinez.nicolas@urjc.es

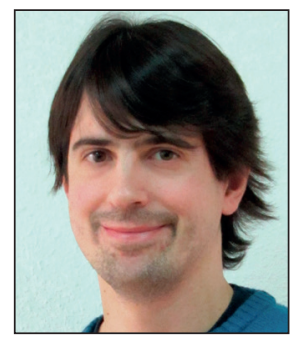

Miguel Vicente-Mariño

https://orcid.org/0000-0002-1467-3535

Universidad de Valladolid

Campus María Zambrano

Plaza de la Universidad, 1

40005 Segovia, España

miguel.vicente@uva.es

\section{Resumen}

Los investigadores en Comunicación formados en España que trabajan en universidades y centros de investigación extranjeros ofrecen un punto de vista aventajado sobre el acceso a la carrera académica en España y el impacto científico internacional de la producción investigadora española. Conocer las razones de su marcha a otros países revela las virtudes y carencias del sistema universitario español. Partiendo de los conceptos habitus (Pierre Bourdieu) y de autopoiesis (Niklas Luhmann), se realizaron entrevistas semi-estructuradas a 13 investigadores elegidos a través de un muestreo de bola de nieve. Los entrevistados centran sus críticas en los mecanismos de selección de profesorado en las universidades españolas, que a su juicio no habrían experimentado una mejora sustancial a pesar de la introducción del vigente sistema de acreditación, generalizado a todas las categorías del profesorado universitario desde hace más de una década, con la implantación del programa Academia en enero de 2008. Los resultados ponen también de manifiesto la pobre imagen de la investigación española en Comunicación en el extranjero, desconectada, salvo excepciones, de las tendencias internacionales en el campo. Las propuestas de mejora identificadas por la muestra consultada, que apuntan a una lenta pero constante incorporación de la investigación española a la esfera internacional, continúan resultando, a juicio 
de las personas entrevistadas, insuficientes para motivar un posible retorno del talento emigrado a las instituciones españolas de educación superior, siendo la insuficiente e intermitente financiación de la investigación uno de los frenos más relevantes. La convergencia con los sistemas que lideran la producción científica mundial en la actualidad se percibe como un escenario improbable y alejado.

\title{
Palabras clave
}

Comunicación; Investigadores; Fuga de talento; Investigación en Comunicación; Endogamia universitaria; Nepotismo; Selección de profesorado; Educación superior; Sistemas de acreditación; Movilidad internacional; Sociología del conocimiento científico; Entrevistas semi-estructuradas; España.

\begin{abstract}
Spanish Communication scholars working at foreign universities offer a vantage point to examine the access to an academic career in Spain and the international impact of Spanish scientific production. The reasons for their departure to other countries lie in the deficiencies of the Spanish university system. Drawing from the concepts of habitus (Pierre Bourdieu) and autopoiesis (Niklas Luhmann), 13 semistructured interviews were conducted with researchers chosen by snowball sampling. The informants highlight faculty hiring mechanisms, which in their view have not improved significantly even after the introduction of an accreditation system in 2008. The results also show the poor image of Spanish Communication research abroad, as it is regarded as disconnected from the international trends in the field. The improvements identified by the consulted sample indicate a slow but steady incorporation of Spanish research into the international sphere. However, this is insufficient, in the opinion of the interviewees, to stimulate a possible return of emigrated talent to Spanish higher education institutions. Insufficient and intermittent research funding is one of the most relevant roadblocks. Convergence with the systems leading the world's scientific production today is still perceived as an unlikely and remote scenario.
\end{abstract}

\section{Keywords}

Communication; Researchers; Brain drain; Communication research; Cronyism; Faculty selection; Higher education; Assessment procedures; Sociology of scientific knowledge; Semistructured interviews; Spain.

\section{Introducción: fuga de talento, también en la investigación sobre Comunicación}

La movilidad laboral ha sido un objeto de interés científico tradicional en diversas disciplinas académicas de las ciencias sociales, destacando entre ellas la economía (Dustmann; Preston, 2019; Hasselbach, 2019) y la sociología (Kuhn; McAusland, 2006; Banas, 2018). Este trabajo se centra en el sector profesional de la investigación y, más concretamente, en el ámbito académico (Doquier; Rapoport, 2012; Appelt et al., 2015; Parey et al., 2017), donde también se han dado contribuciones significativas que analizan la movilidad del capital humano y su impacto en el sistema científico y universitario desde un punto de vista bibliométrico (Deville et al., 2014; Albarrán; Carrasco; Ruiz-Castillo, 2017; Carrasco; Ruiz-Castillo, 2019).

La evidencia empírica acumulada en estos trabajos se ha canalizado a través de conceptos que remiten a la fuga de cerebros (elite brain drain), a la circulación de conocimiento (brain circulation) o a la atracción de talento (brain gain). Estos términos apuntan al hecho de que el potencial científico se ha convertido en una valiosa mercancía para las universidades y los centros de investigación, entidades que se hallan inmersas en una lógica de competencia por el liderazgo científico en diferentes escalas, desde la estatal hasta la global. La capacidad de instituciones y países para atraer talento actúa como un indicador de su potencial en investigación, desarrollo e innovación.

En febrero de 2017, la Fundación Española para la Ciencia y la Tecnología (FECYT) celebró en un artículo (Elorza-Moreno et al., 2017) el valor de la diáspora científica española como representante excelsa de la 'marca España'. Los científicos españoles en el extranjero formarían una red que atestigua la calidad de la ciencia española y, al mismo tiempo, ejercerían cierta función de 'diplomacia pública', conectando a España con los centros de referencia en investigación. Este tono celebratorio ofendió a algunos de los miembros de esa misma red. En un artículo publicado en Nature, la astrofísica española Amaya Moro-Martín, radicada desde 2014 en el Space Telescope Science Institute en Baltimore (EUA), replicaba que si algo evidenciaba la red de científicos españoles en el extranjero eran las condiciones precarias de la ciencia en España que motivaban la fuga de cerebros (Moro-Martín, 2017).

Es difícil cuantificar la diáspora científica española. A falta de un censo oficial, las fuentes más fiables son las propias asociaciones de científicos españoles en el extranjero. Según el presidente de la Sociedad de Científicos Españoles del Reino Unido (Ceru), Eduardo Oliver, los tres países en los que se concentra la mayoría de la diáspora investigadora española son Estados Unidos, Reino Unido y Alemania (López-Rejas, 2016). Si sumamos los miembros de las asociaciones de científicos españoles en Estados Unidos (Españoles Científicos en USA, Ecusa, unos mil miembros), Reino Unido (550 indivi- 
duos), Alemania (Sociedad de Científicos Españoles en la República Federal de Alemania, Cerfa, 600 suscriptores) y Australia-Pacífico (Spanish Researchers in Australia-Pacific, SRAP, medio centenar de miembros), la cifra ascendería a 2.200 científicos. Sin embargo, la red de asociaciones Raicex afirma representar a 17 agrupaciones con más de 4.000 investigadores y científicos españoles en el exterior. Esta última cifra quizá sea aún conservadora, ya que las asociaciones mencionadas aglutinan sobre todo a investigadores de las ramas científico-tecnológica y biosanitaria, y se carece de datos fiables relativos a las ciencias sociales y las humanidades.

Si bien el estado de la ciencia española nunca ha sido boyante en términos comparativos (la inversión en ciencia se sitúa en un máximo del 1,4\% del PIB, mientras que la media europea es del 2\%), el estallido de la crisis económica de 2008 supuso una drástica reducción del presupuesto para innovación y desarrollo (de 2009 a 2013 cayó un 39\%, y siguió sin remontar hasta 2017). Según la Fundación Cotec (2019), todavía en 2019 el sector público de I+D (universidades y centros de investigación) registraba 8.660 empleados menos que en 2010 (Segarra, 2019). No es casualidad, pues, que las asociaciones de científicos españoles en la diáspora se fundaran, precisamente, coincidiendo con la situación generada por la crisis (Ceru en 2012, Cerfa en 2013, Ecusa en 2014).

Los estudios de Albarrán, Carrasco y Ruiz-Castillo (2017) y Carrasco y Ruiz-Castillo (2019), limitados al espacio europeo en el campo de las Ciencias Económicas, concluyen que España cuenta con una mayor capacidad de atracción de talento y de circulación de conocimiento, por lo que las dimensiones de la fuga de cerebros en esa área es menor que en otros sistemas europeos de dimensiones y características similares, como Alemania, Francia o Italia. Los trabajos centrados en la investigación en Ciencias de la Salud (García-Romero, 2012; Aceituno et al. 2015; Aceituno et al., 2017; Aceituno et al., 2018) son también abundantes. Con datos obtenidos mediante una encuesta $(\mathrm{N}=811)$ a investigadores españoles del sector de la salud que trabajan fuera de España,

Aceituno et al. (2017) revelan que los principales incentivos para marcharse al extranjero fueron la posibilidad de mejorar la carrera investigadora y la formación, de obtener financiación para investigar, y de incrementar el salario. Ya en 2013, un proyecto de periodismo de datos impulsado por El periódico de Catalunya encuestó a 400 científicos españoles en el extranjero (Catanzaro, 2013), y solo uno de cada diez entrevistados consideraba la posibilidad de volver a España. En el extranjero encontraban sueldos superiores, financiación para sus proyectos y la posibilidad de contribuir a las líneas de investigación científica internacionalmente más relevantes desde el centro o, por expresarlo en términos de una división Norte-Sur, desde el “Norte Global” (Demeter, 2019).

En el campo de la Ciencia Política, quizá más cercano al de la Comunicación, Afonso (2016) sitúa a España, Francia e Italia como ejemplos de mercados académicos cerrados (esto es, "hostiles" para los candidatos no locales) y potencialmente seguros (la carrera académica termina habitualmente en la adquisición de la condición de funcionario). Más abiertos, pero igualmente capaces de ofrecer seguridad laboral, serían a su juicio Reino Unido, Países Bajos y Escandinavia. Suiza sería un país abierto, pero que no ofrece muchos contratos permanentes, mientras que Alemania sería un país cerrado y con una mayoría de la fuerza laboral con contrato temporal. Sin duda, cabría matizar la seguridad laboral que Afonso atribuye al mercado académico español, habida cuenta de la creciente precarización del empleo universitario en España.

Si obtener datos fiables de la diáspora científica española en general es difícil, especificar el número de investigadores en Comunicación en el extranjero lo es todavía más. Pero los hay. Es más, algunos de ellos (entrevistados para este trabajo) ocupan posiciones de alta responsabilidad en centros de referencia en sus respectivos países de acogida. Rondan la cuarentena y se encuentran en su momento de 'peak performance', de mayor productividad. Se encuentran, pues, en una posición de privilegio para comparar la investigación en comunicación en España con la que se realiza en el Norte Global. Ese es uno de los objetivos de esta investigación, además de conocer las razones de su marcha y tomar nota de sus 'lay theories' o explicaciones propias para dar sentido a su propia condición de expatriados científicos, prestando también atención a sus propuestas de solución o mejora.

El interés en la comunidad científica de los estudiosos de la Comunicación en el extranjero viene motivado por el propio crecimiento del gremio. Desde los años 90, la oferta de titulaciones de Comunicación en España ha aumentado de manera espectacular. Si en el cambio de milenio los alumnos que querían ingresar en estas titulaciones tenían apenas un puñado de opciones en el sector público (Madrid, Barcelona, Bilbao, Tenerife, Santiago de Compostela, Málaga, Sevilla), en 2015 había ya 54 universidades ofreciendo titulaciones de Comunicación, que contaban con unos 4.000 docentes/investigadores (Saperas, 2016). La elaboración de un censo fiable continúa siendo una tarea que, a pesar de su dificultad, convendría acometer de cara a un mejor conocimiento de esta comunidad investigadora.
En 2019 el sector público de I+D (universidades y centros de investigación) registraba 8.660 empleados menos que en 2010 
La gran recesión que tanto afectaría al presupuesto público de ciencia coincidió con la implantación en 2008 del programa Academia de acreditación del profesorado universitario por parte de la Agencia Nacional de Evaluación de la Calidad y Acreditación (Aneca), con exigentes requisitos en cuanto a productividad científica. En el bienio 2011-2012, una batería legislativa limitó la tasa de reposición de empleados públicos jubilados, lo que generó un cuello de botella que ayudaría al éxodo científico de jóvenes académicos sin perspectiva laboral (Arenilla-Sáez, 2012; Amoedo-Souto; Nogueira-López, 2013). Incluso aquellos que consiguieron pasar el filtro meritocrático de la acreditación de la Aneca veían cómo se retrasaba sine die su acceso a las categorías del funcionariado docente. Como atestigua la experiencia de los investigadores entrevistados para este trabajo, se hacía inevitable una fuga de talento que también afectaría a los investigadores en comunicación.

El estudio de las trayectorias vitales de los investigadores que desarrollan sus carreras profesionales en el extranjero es relevante para un abordaje sociológico de la producción del conocimiento científico, y debe ser también un foco de atención para las políticas científicas. En este sentido, los programas de recuperación del talento puestos en marcha por las administraciones públicas, de alcance tanto estatal (Juan de la Cierva, Beatriz Galindo, Ramón y Cajal) como autonómico (Icrea, Atracción de Talento, Ikerbasque...) ponen de manifiesto la importancia de recuperar unos perfiles que han demostrado contar con capacidad suficiente como para desarrollar parte de sus carreras en sistemas académicos extranjeros.

\section{Perspectiva teórica: habitus y autopoiesis}

Uno de los académicos pioneros en el estudio de las desigualdades Norte-Sur en la investigación en Comunicación, Demeter (2019), utiliza el marco conceptual propuesto por Bourdieu (2008) para la sociología de la ciencia y del conocimiento científico. Los académicos trabajarían en un 'campo profesional' que gozaría de una serie de reglas internas, cuya asimilación como hábitos por parte de los científicos (el 'habitus') daría lugar a una acumulación de mayor 'capital' académico. Esta perspectiva teórica es plenamente válida para nuestro objeto de estudio, el establecimiento de las causas de la diáspora científica española en el campo disciplinar de la Comunicación. Existiría pues un "Norte Global", fundamentalmente anglosajón, que marcaría las pautas del campo: las universidades de referencia, las revistas académicas más relevantes..., y por extensión las teorías y métodos considerados válidos u óptimos dentro de la comunidad científica. Frente a este, el "Sur Global" estaría formado por académicos que tratarían de engancharse a ese discurso dominante estableciendo filiaciones con académicos del Norte, o con la creación de sus propios nichos académicos mediante asociaciones regionales o cadenas alternativas de publicación.

La movilidad internacional es, según Demeter (2019), uno de los aspectos clave en la construcción de un 'habitus' competitivo para los académicos del Sur Global. Tras entrar en contacto con las instituciones del Norte (por ejemplo, un posgrado en una universidad estadounidense), el científico interioriza una serie de pautas y rutinas, tales como la retórica de un paper persuasivo, los métodos y teorías en boga, los journals en los que merece la pena publicar o los congresos donde pueden hacerse los mejores contactos. A este respecto, los investigadores entrevistados para este trabajo reconocerán que el salto internacional consiste en gran medida en la internalización de ese 'habitus'.

El 'habitus' del Norte Global no coincide necesariamente con el del Sur. Todo profesor del Norte recibe un 'welcome package', un paquete de bienvenida, en el que se le explican las características básicas de la universidad que lo acoge. Dicho paquete sería innecesario en España, pues en la mayoría de ocasiones el profesor que consigue una plaza ha conocido su institución como estudiante. Manuales como el de Perlmutter (2010) sobre cómo moverse en el mercado académico anglosajón codifican reglas ajenas para el científico español: no hay en España viajes pagados por los departamentos para que el candidato se someta a entrevistas e imparta clases de prueba; no existe el ofrecimiento del pago de los costes de una mudanza, etc. En definitiva, no existe un mercado académico como tal, en el que las universidades compitan por hacerse con los servicios del recién doctorado más prometedor.

Junto a Bourdieu, otro referente teórico clave a la hora de explicar las posibles razones del éxodo científico español, y el de la investigación sobre comunicación en particular, es el del sociólogo alemán Luhmann (1997) y su teoría de los sistemas sociales. En concreto, es de gran valor su concepto de 'autopoiesis', la idea de que los sistemas complejos llegan a cobrar una lógica propia que es entendible solo para los 'insiders', esto es, sus propios partícipes. Así, mientras que en Estados Unidos el proceso de contratación de profesores puede ser relativamente análogo al de cualquier otra profesión liberal (se toma en consideración la trayectoria curricular, se entrevista al candidato, y se le somete a una prueba simulatoria -una clase o la presentación de un paper-), el sistema español, deudor de la tradición del derecho administrativo, requiere del aspirante dos pasos: una habilitación nacional (acreditación Aneca) y un concurso-oposición bajo los términos de la universidad convocante de la plaza, que en la mayoría de los casos cuenta con un candidato local como preferencia no explícita.
Incluso aquellos que consiguieron pasar el filtro meritocrático de la acreditación de la Aneca veían cómo se retrasaba sine die su acceso a las categorías del funcionariado docente 
Evidencias del carácter autopoiético del sistema universitario español serían su alto grado de endogamia (Marín; Álvarez, 2014, estiman que el 73\% de los profesores lo son de la misma universidad donde obtuvieron su doctorado), su burocratización (solicitud de certificaciones de toda actividad susceptible de considerarse un mérito, desde un curso de ofimática al número de horas que los estudiantes han utilizado un sistema virtual de gestión del aprendizaje) y rigidez administrativa (la costumbre estadounidense de no presentarse a una plaza ofrecida por la universidad en la que se obtuvo el doctorado iría contra el derecho de todo ciudadano a ejercer la función pública en España).

La autopoiesis del sistema distancia a sus 'insiders' del resto de la sociedad. José Carlos Bermejo, catedrático de la Universidad de Santiago de Compostela y una de las voces más críticas con el sistema universitario español, considera que

"las universidades, gremios que defienden básicamente los intereses de sus profesores, en menor medida de su personal administrativo y prácticamente nada de sus alumnos, viven también cada vez más al margen de la realidad",

de tal manera que el saber estaría dejando los campus para trasladarse a las empresas, sometiendo el conocimiento al albur de

“los grandes intereses económicos, políticos o militares" (Bermejo, 2009).

El también catedrático Félix De-Azúa llega incluso a afirmar que la universidad "es una de las instituciones más corruptas del conjunto institucional español", con "tribunales amañados", "convocatorias a medida" y "planes de estudio deformados departamento a departamento, según el interés de la plantilla” (De-Azúa, 2014).

La inacción ante los recientes escándalos como el de las tesis plagiadas en una universidad privada española, sin respuesta por parte de la Conferencia de Rectores de Universidades Españolas (CRUE) o de la propia universidad, que podría haber retirado el doctorado a los sospechosos, vendría a reforzar la idea de un sistema con su propia lógica, en el que el teórico garantismo del derecho público se revela inútil ante el corporativismo. A decir de Pablo Jauralde-Pou, catedrático de la Universidad Autónoma de Madrid,

"el sistema universitario ha generado, como todas las estructuras de poder, sus propias reglas de juego: nadie que esté fuera del sistema puede criticarlo, y si lo hace, será descalificado" (Jauralde-Pou, 2008).

Aunque el estudio de Demeter (2019) apunta hallazgos valiosos (como el hecho de que, para publicar en journals internacionales, lo mejor que puede hacer un académico del Sur Global es aliarse con un académico del Norte Global o, en su defecto, aquilatar su capital académico estudiando un posgrado en ese mismo Norte), la bibliografía existente no nos permite aventurar predicciones en forma de hipótesis. En su lugar, se proponen tres preguntas de investigación que orientaron las entrevistas con los investigadores españoles en Comunicación de la diáspora:

PI1. ¿Qué razones motivan a los investigadores españoles en Comunicación a buscar una carrera académica en el extranjero?

PI2. ¿Cuál es la imagen que estos investigadores tienen de la producción científica en Comunicación realizada desde España, y la impresión que a su juicio tiene entre sus colegas extranjeros?

PI3. ¿Qué propuestas de reforma ofrecen estos investigadores para mejorar el sistema universitario español y la investigación en Comunicación en España?

\section{Método: entrevistas semiestructuradas}

Las entrevistas semiestructuradas son el método que mejor se adapta al objetivo de conocer la experiencia de los académicos españoles en el extranjero. Siguiendo las tres preguntas-marco arriba señaladas, el cuestionario se orientó además a obtener pequeñas historias de vida a fin de poder extraer de ese discurso biográfico aspectos relevantes que podríamos no haber anticipado en el diseño de la batería de preguntas elaborada previamente (Weiss, 1994). El enfoque cualitativo del planteamiento de esta investigación encaja también con el carácter exploratorio de la misma, ya que, hasta donde tenemos conocimiento, este es el primer trabajo en abordar las razones de la diáspora científica española en el campo disciplinar de los estudios de Comunicación.

Carentes de un censo de investigadores en el extranjero, y teniendo en cuenta que las asociaciones de científicos españoles en Estados Unidos, Reino Unido y Alemania agrupan sobre todo a académicos de las ramas científico-tecnológica y bio-sanitaria, optamos por un muestreo de bola de nieve. Contactamos en primer lugar a destacados colegas que conocíamos en persona o por sus publicaciones y les pedimos que nos indicaran otros académicos en situación análoga. El trabajo de campo comenzó en la primavera de 2016, y se prolongó hasta finales de 2019. 
El llamado 'punto de saturación', momento en el cual los informantes no añaden información nueva que sea de interés para la investigación, se constató al alcanzar la docena de entrevistas. El perfil de nuestros participantes, 8 varones y 5 mujeres, reparte su destino geográfico entre Reino Unido, Estados Unidos y la Europa continental. Todos los entrevistados se encuentran entre la treintena y la cuarentena, pero la duración de su estancia en el extranjero varía enormemente, con casos que van desde los 2 a los 23 años (véase la tabla 1).

Las entrevistas se realizaron por vídeo-conferencia y se grabaron en audio para su posterior transcripción. El análisis temático, esto es, la identificación de temas emergentes en el discurso de los informantes, estuvo asistido por un software para el análisis de textos cualitativos, TAMS Analyzer, que permite etiquetar y relacionar los temas-clave de cada una de las transcripciones.
Tabla 1. Perfil de los entrevistados

\begin{tabular}{|l|c|l|c|}
\hline \multicolumn{1}{|c|}{ Sexo } & Edad & País de residencia & Años en el extranjero \\
\hline Varón & 37 & Reino Unido & 10 \\
\hline Mujer & 31 & Reino Unido & 2 \\
\hline Mujer & 37 & Reino Unido & 6 \\
\hline Varón & 39 & Reino Unido & 9 \\
\hline Varón & 46 & Estados Unidos & 11 \\
\hline Varón & 41 & Estados Unidos & 23 \\
\hline Mujer & 45 & Estados Unidos & 9 \\
\hline Mujer & 38 & Estados Unidos & 16 \\
\hline Mujer & 42 & Estados Unidos & 7 \\
\hline Varón & 41 & Bélgica & 4 \\
\hline Varón & 45 & Dinamarca & 12 \\
\hline Varón & 42 & Suecia & 4 \\
\hline Varón & 37 & Suiza & 8 \\
\hline
\end{tabular}

A fin de garantizar la confidencialidad de los entrevistados, se optó por registrar únicamente su sexo, edad, país de destino y número de años fuera de España. Se ha evitado además seleccionar citas que pudieran, por el tipo de personas o instituciones que mencionan, facilitar la revelación de la identidad del entrevistado.

\section{Resultados}

\subsection{Motivaciones para emprender una carrera científica en el extranjero}

\subsubsection{Nepotismo y endogamia}

Si admitiéramos que la selección del profesorado universitario en España es justa, llegaríamos a la conclusión de que no existe un problema de retención de talento. Tras aplicarse rigurosísimos controles y una objetiva valoración de los méritos de cada candidato, casualmente tres de cada cuatro aspirantes se han doctorado en la universidad en la que pretenden ejercer la docencia y practicar la investigación (Marín; Álvarez, 2014). El sistema universitario español tendría la enorme "suerte" de encontrar a los mejores en casa. Sorprendentemente, otros países de la Unión Europea no son tan afortunados. En Reino Unido y Alemania el porcentaje de retención del talento doméstico es del 8\%. En Italia se eleva al 24\%, un índice de retención alto, pero todavía no comparable al español (Caballé, 2014).

Los investigadores entrevistados entienden que esas tres cuartas partes de docentes que trabajan en la misma universidad en la que se doctoraron no están ahí por ser los mejores en su campo, sino por estar mejor conectados con el poder local, entendiendo por tal al propio departamento, a un catedrático de referencia o a otros miembros del gobierno universitario:

“Mi salida de España viene principalmente motivada por el contexto académico español: nepotismo, plazas dadas, deber favores, callar para que se te contrate..., por la constatación de que no encajaba académicamente. Me resultaba difícil entender que personas menos capacitadas que yo me pasaran por delante: una vez era porque llevaban mucho tiempo de asociados, porque tenían un padrino más poderoso, porque tenían hijos... [...]. El tema de la 'plaza dada' es un drama: limitas la competencia al ganador, y es muy frustrante para el resto" (Varón, 37 años, Reino Unido, 10 años en el extranjero).

“En Estados Unidos la meritocracia funciona. Todo depende de mí, a diferencia de España, donde hay demasiadas cosas que escapan a tu control. Todos necesitamos un mentor, también en Estados Unidos, pero no un padrino, que es lo que se necesita en España" (Mujer, 42 años, EUA, 16 años en el extranjero).

Uno de nuestros informantes apunta que el teórico umbral de excelencia que marcaría la acreditación de la Aneca no es suficiente, ya que entre candidatos acreditados en liza siempre ganará el candidato señalado como favorito por el departamento:

"La endogamia lo que hace es que al final no contrates a los investigadores más cualificados, sino que a los investigadores que llevan más tiempo o que pertenecen a un determinado círculo. Esto mejoró un poquito con la puesta en marcha de las agencias de evaluación, pero al final lo que estas hacen es marcar una serie de mínimos, y no garantizan un sistema de selección por excelencia. Hay candidatos que renuncian a competir por una plaza cuando ven que los miembros del tribunal tienen una relación más que directa con el candidato local. No hay un fair play, no todos jugamos con las mismas cartas ni con las mismas posibilidades" (Varón, 37 años, Suiza, 2 años y medio en el extranjero). 
La sensación de un sistema injusto, en el que la libre competencia no está garantizada, desincentiva a candidatos de talento desconectados de las estructuras de poder local, que suele ser el caso de los residentes en el extranjero. Al parecer de nuestros informantes, siendo el nepotismo una práctica extendida en todo el sistema universitario español, no existe la posibilidad de salvar la endogamia presentándose como candidato en otra universidad del Estado. La única opción viable es el extranjero y, más concretamente, el Norte Global. Es más, paradójicamente, pese a los requisitos de la Aneca al respecto, la movilidad no ayuda, sino que reduce las posibilidades de ser contratado como docente universitario en España (Aunión, 2013).

\subsubsection{Falta de perspectivas profesionales}

Mientras que en países como Estados Unidos los doctorandos suelen disfrutar, como norma general, de una beca que garantice su subsistencia, en España el doctorando becado es la excepción y no la regla. A ello hay que sumar las restricciones presupuestarias derivadas de la Gran Recesión, que han limitado las posibilidades de promoción académica. En el curso 2016-2017, dos tercios de los profesores que accedían a la condición de funcionario (profesor titular de universidad) superaban los 50 años (Álvarez; Vázquez, 2018). En los Estados Unidos, donde los programas de doctorado son más largos al incluir al menos dos años de docencia antes de iniciar la tesis, la edad media de la titularidad ('tenure') se sitúa en los 39 años (Academic Careers Observatory, 2018).

Ante estos condicionantes (doctorados no financiados y escasas expectativas de promoción), para algunos de los entrevistados la única vía para proseguir una vida académica se encontraba fuera de España:

"Con la crisis, en España se taponaron los sistemas de promoción sine die. Se acababa mi beca posdoctoral y la plaza de [profesor] titular para la que estaba acreditado hacía tiempo no iba a salir nunca, por lo que las posibilidades se cerraban mucho a nivel académico" (Varón, 37 años, Suiza, 2 años y medio en el extranjero).

"Los salarios españoles son un tercio de lo que se puede ganar aquí" (Varón, 46 años, EUA, 9 años en el extranjero).

“En España no conseguía ninguna beca FPU o FPI, pero en el Reino Unido me becó la universidad a la que solicité admisión para hacer un máster" (Varón, 37 años, Reino Unido, 10 años en el extranjero).

La eclosión de posgrados en Comunicación de la universidad española se produce sin unas garantías de financiación para los doctorandos. Compárese esta situación con la decisión de universidades como la George Washington University de Estados Unidos. A pesar de contar en su plantel de profesores con académicos de primer rango, como Robert M. Entman, una de las referencias mundiales en la investigación sobre framing, o Silvio Waisbord, editor del Journal of communication entre 2015 y 2019, su School of Media and Public Affairs no ofrece un doctorado porque no ha encontrado la manera -o no considera una prioridad- de financiar a todos sus estudiantes de tercer ciclo. Por parafrasear las palabras de uno de sus directivos a uno de los autores de este trabajo: si no hay dinero para financiar a todos los estudiantes, el programa no se abre.

\subsubsection{Ambición personal}

La investigación sobre los fenómenos migratorios revela que los emigrantes suelen gozar de un plus de ambición y valentía sobre los que se quedan en el país de origen (The economist, 2011). La diáspora científica española, también en investigación de Comunicación, presenta un fenotipo que prioriza la libertad sobre la seguridad, el riesgo sobre la tranquilidad:

“Confluyeron la inquietud vital por conocer otras realidades con ese tapón de la academia [española]. Entonces me dije: 'Bueno, vamos a probar suerte fuera, a ver qué otras realidades hay ahí'” (Varón, 37 años, Suiza, 2 años y medio en el extranjero).

“Estuve trabajando muchos años en consultoría, y aquello no me llenaba [...]. Cuando acabé mi doctorado, cansado de hacer lo mismo durante 11 años, me decidí a tramitar mi número de la Seguridad Social en la Embajada de los Estados Unidos, en Madrid" (Varón, 46 años, EUA, 9 años en el extranjero).

\subsection{Opinión sobre la investigación en Comunicación realizada en España}

\subsubsection{El inglés no es el problema}

Una de las excusas más recurrentes en la academia española para justificar la escasez de su presencia en publicaciones internacionales o la limitada movilidad de sus miembros es el papel dominante del inglés en la comunicación científica. El problema no sería la calidad o la orientación de la ciencia social o humanística que se practica en España, sino que el handicap radicaría en el infortunio de no ser un hablante nativo de la lengua franca global.

La mayoría de los entrevistados lo niega. A su juicio, el problema está en las teorías, los métodos y las fuentes empleadas, que en muchos casos se limitan al discurso académico nacional, sin entrar en diálogo con la investigación internacional:

"Es más una cuestión de fondo, no es una cuestión lingüística. Los investigadores españoles recurren a traductores profesionales, por lo que el inglés del artículo puede ser estupendo, pero falla todo lo demás. Falta conexión 
con la bibliografía internacional. Lo que hay son referencias a la gente que va a estar en los tribunales [españoles]. También abundan referencias de 1996 para sostener una afirmación sobre Facebook, o un montón de expresiones que usamos los latinos ('es indudable que', 'no cabe duda de que') que para un anglo chirrían mucho. ¿Cómo que es indudable? ¿Quién lo dice? ¿Tienes evidencia para demostrarlo?” (Varón, 37 años, Reino Unido, 10 años en el extranjero).

"En la academia española no es extraño encontrarte un paper que esté publicado sin ninguna referencia internacional, solo a cosas escritas en castellano [...]. Entonces, no se trata simplemente de traducir el texto al inglés. Se trata de las fuentes que se manejan, se trata del rigor de las metodologías empleadas, se trata en muchos casos de las teorías empleadas..., que no son de referencia porque, a lo mejor, son de otros investigadores españoles que no se conocen fuera del propio país" (Varón, 37 años, Reino Unido, 10 años en el extranjero).

“Mi impresión es que [la investigación sobre Comunicación en España] va 20 años por detrás. Lo que se investiga no interesa. En mi experiencia como evaluadora de proyectos del Ministerio [Plan Estatal de I+D], veo que lo que se presenta son propuestas con temas muy locales y poco conectadas con la investigación de frontera. Son casi trabajos de consultoría. Falta conocer dónde está la frontera actual del conocimiento. Las teorías son quizá también un tanto anticuadas" (Mujer, 42 años, EUA, 16 años en el extranjero).

\subsubsection{Predominio del humanismo sobre el empirismo}

La investigación sobre Comunicación se debate disciplinariamente entre dos mundos, el de las humanidades, tradicionalmente más cualitativas y ensayísticas, y el empirismo de la ciencia social, con un predominio del método cuantitativo y sus técnicas, y guiado por el razonamiento hipotético-deductivo. La producción científica de los académicos españoles, a decir de los investigadores entrevistados, se sitúa claramente en el marco cualitativo-humanístico, minoritario en el discurso académico internacional:

“Quizá la investigación española es poco empírica para los estándares que se manejan a nivel internacional [...]. En Estados Unidos estoy acostumbrado a un nivel de especialización que en España no veo. En la mayor parte de los casos, los grupos de investigación españoles producen estados de la cuestión, pero 'there's no finding', que se diría por estos lares" (Varón, 46 años, EUA, 9 años en el extranjero).

“Francia es todavía más insular que España [...]. Su investigación es muy humanística, por lo que están bastante desconectados de la hegemonía empirista que domina en las conferencias internacionales" (Varón, 37 años, Reino Unido, 10 años en el extranjero).

La investigación sobre Comunicación, como todas las disciplinas científicas sociales, es pluriparadigmática, y no es infrecuente que un mismo autor navegue entre las corrientes funcionalista, hermenéutica y crítica (Montero-Sánchez, 1994). Pero la menor producción de ciencia cuantitativa en el campo de la Comunicación quizá se deba a la falta de un profesorado formado en técnicas estadísticas. Mientras que en titulaciones como Psicología, Sociología o Ciencia Política la formación estadística es común a todos los graduados, en las titulaciones de Comunicación la estadística puede llegar a ser, si acaso, una asignatura optativa. Esta carencia no se soluciona en los másteres de investigación en comunicación, cuyas asignaturas suelen responder al expertise temático de los docentes.

\subsubsection{Vieja academia vs. Nueva academia}

A pesar de su aparente desconexión de la academia del Norte Global, la academia española actual comparte un rasgo común, no necesariamente positivo, con la ciencia internacional: la obsesión por el 'publish or perish'. El académico de la vieja escuela podía permitirse el lujo de considerar la publicación de su tesis en forma de monografía en una editorial local como el gran logro profesional de su carrera. El joven académico actual sabe que su acreditación y promoción profesional están condicionadas por la publicación en revistas de alto impacto. Y aunque el Sur Global dispone ya de journals en español que gozan de buena posición en los rankings (véanse los casos de Comunicar

Para publicar en journals internacionales, lo mejor que puede hacer un académico del Sur Global es aliarse con un académico del Norte Global

y de Profesional de la información, revistas editadas en España indexadas en los prestigiosos Journal Citation Reports), el académico de nuevo cuño tiene la necesidad de entrar a formar parte del discurso internacional liderado por el Norte Global. Debe, pues, trascender la insularidad de sus fronteras nacionales y engancharse a las teorías, métodos y rutinas (al 'habitus') del paradigma dominante, el anglosajón.

El sistema académico español reproduce así la famosa dualidad que se atribuye al mercado laboral nacional: un cuerpo funcionarial que goza de seguridad laboral y que pudo auparse hasta su posición compitiendo en un escenario fundamentalmente nacional, frente a un precariado obligado a encadenar contratos temporales pero que consigue publicar allí donde su mentor-catedrático jamás lo hará, y que se mueve cual pez en el agua en congresos internacionales que serían un ambiente extraño e incluso hostil para sus 'superiores' en la jerarquía académica:

"Hay como dos academias dentro del ámbito de la Comunicación. Por un lado, veo la academia más tradicional, que se mueve con las reglas que le han funcionado hasta ahora: hacer investigación para un público nacional, financiada en el ámbito nacional, publicada en el ámbito nacional. Ahí están la mayoría de IP [investigadores 
principales] españoles. Luego está ese grupo que viene de una cultura más nueva, gente más joven que, de forma aislada, ha dado el salto al ámbito internacional [...]. Son investigadores junior que se ven obligados a publicar en revistas internacionales para promocionar" (Varón, 37 años, Suiza, 2 años y medio en el extranjero).

\subsection{Soluciones sugeridas para mejorar el rendimiento investigador español}

\subsubsection{Impedir contratar a doctores de la propia universidad}

A pesar de que no existe una legislación federal que justifique poder hablar de un 'modelo estadounidense de universidades', es conocida la costumbre -que no ley- de que no se contrate a un doctor que ha leído su tesis doctoral en la universidad que convoca una plaza. La tradición se remonta a 1909, cuando Charles W. Elliot, rector de Harvard, decidió adoptar dicha medida para atajar la endogamia (Marín; Álvarez, 2014).

Los investigadores entrevistados ven con buenos ojos la 'política Elliot' y se muestran partidarios de trasladarla al contexto español:

"Esto es muy fácil: si tú tienes el título por la Universidad de Pepito, no puedes estar empleado en la Universidad de Pepito. Te tienes que ir a otra universidad. Punto" (Mujer, 45 años, EUA, 23 años en el extranjero).

"El mundo académico de aquí [EUA], por lo que yo he visto, es mucho menos endogámico que el de España. Aquí, por ejemplo, es muy raro que una persona que haga un doctorado en una universidad se quede trabajando en el mismo centro. Lo echan a patadas. Me parece muy bien: hay que renovar" (Varón, 41 años, EUA, 11 años en el extranjero).

Sin embargo, adoptar una política anti-endogámica como la americana atentaría contra el derecho de todo ciudadano español a acceder a la función pública. Ello no ha impedido que las facultades de Económicas de universidades como la Autònoma de Barcelona, Pompeu Fabra, Carlos III de Madrid y Alicante hayan adoptado el modelo estadounidense mediante la creación de un job market. Desde 1995, y a imitación del congreso anual de los economistas estadounidenses, el simposio de la Asociación Española de Economía celebra una feria de empleo en la que universidades y empresas entrevistan a los recién doctorados (Marín; Álvarez, 2014). Estos deben preparar lo que convencionalmente se conoce como un 'job market paper', una presentación habitualmente basada en su tesis doctoral que será valorada por los posibles empleadores.

Otra vía para atraer académicos de excelencia, vista la rigidez del aparato administrativo español, es la creación de sistemas paralelos al ordinario: los programas Icrea en Cataluña, Ikerbasque en el País Vasco e Imdea en Madrid tienen precisamente el objetivo de atraer el talento que escapa a las rigideces burocráticas del funcionariado.

\subsubsection{Portal de empleo académico centralizado}

Las universidades españolas tienen la extraña "virtud" de encontrar casi todo el talento que necesitan dentro de sus propios recintos, y de ahí que la necesidad de publicidad de los puestos de trabajo en oferta sea mínima. Mientras que cualquier interesado puede crear alertas o buscar online ofertas de empleo académico sea en Estados Unidos (Chronicle Vitae), el Reino Unido (Academic Jobs UK) o los Países Bajos (Academic Transfer), el aspirante español ha de estar atento a los boletines oficiales del Estado y las Comunidades Autónomas, cuando no a las webs de las propias universidades.

En España llegó a existir algo parecido a un portal centralizado de empleo académico en la web del Ministerio de Educación, pero las escasas ofertas que llegaban a publicarse (una exigua fracción de las que se convocaban) requerían la consulta de documentos previo conocimiento del código Unesco de la disciplina en cuestión. No es de extrañar, pues, que la ausencia de un portal de empleo académico de referencia sea una de las demandas de los investigadores españoles en el extranjero:

"Cuando me pongo a buscar en internet ofertas de empleo de universidades españolas... primero, no hay un sitio centralizado donde tú encuentres qué plazas se están ofreciendo. En Estados Unidos hay tres o cuatro webs donde viene todo" (Mujer, 45 años, EUA, 23 años en el extranjero).

Una conjetura razonable es que no interese la publicidad de las plazas a fin de garantizar el acceso al candidato local sin mayores contratiempos. De hecho, en el sistema español se considera que el triunfo de un candidato externo (altamente improbable en todo caso, ya que se procura que los miembros del tribunal evaluador sean de plena confianza) representa una merma económica para el departamento convocante, pues, además de asumir el 'fichaje' foráneo, probablemente haya de proveer una nueva plaza para el candidato local perdedor.

\subsubsection{Desfuncionarización de la docencia y la investigación}

El carácter funcionarial de las figuras permanentes parece ser uno de los principales obstáculos para la captación de talento internacional. Las universidades no tienen margen para hacer ofertas suculentas a un candidato excelente, ni pueden privar de acceder a la función pública a un candidato local. Desde el punto de vista del derecho administrativo español, entrevistar a candidatos es incluso problemático, ya que supone introducir un sesgo de subjetividad en un proceso que se presume ciego, donde los méritos objetivables deben ser el principal y casi único elemento de valoración. La rigidez del aparato administrativo es uno de los principales problemas del modelo español, según los investigadores entrevistados: 
“El problema es que la ventaja de la funcionarización -la libertad del profesorado y su blindaje frente a los cambios políticos- ha devenido en impunidad" (Varón, 37 años, Reino Unido, 10 años en el extranjero).

"El sistema tiene que ser más permeable. En España tenemos un sistema de oposición complejo que blinda las plazas para la gente que ya está dentro. Sería bueno que los departamentos tuviesen autonomía para contratar a quien quisieran [...]. No hay sistema de incentivos: hay una tabla rasa salarial" (Varón, 46 años, EUA, 9 años en el extranjero).

En las entrevistas realizadas, y también en el discurso periodístico, se utiliza a menudo la analogía del fútbol: la captación del talento es difícil desde un marco funcionarial. Se necesita flexibilidad para recompensar a la persona excelente. Pero, ¿cómo hacerlo desde un sistema público? De nuevo, el sistema establecido no ofrece soluciones. Se ha planteado la figura de un profesor visitante cuyo salario vendría determinado por las Comunidades Autónomas o por el Consejo Social de la universidad concernida (Silió, 2019). Otra vía son los programas públicos de captación de talento Ramón y Cajal y Juan de la Cierva, pero estos tampoco resultan convincentes a nuestros entrevistados:

"Los salarios de esos programas están entre los 28.000 y los 36.000 euros al año. No son competitivos. Pero es que además del salario, el investigador necesita aterrizar en un entorno en el que haya dinero para llevar a cabo los proyectos. Cuando la inversión en investigación no es una prioridad, lo que se prometía como un contrato renovable a cuatro años se queda en dos. No puedes hacer eso con alguien que ha dejado Estados Unidos para venirse a España" (Mujer, 31 años, Reino Unido, dos años en el extranjero).

"La verdad es que no me planteo volver a España. La única posibilidad sería a través de plazas fijas financiadas por el gobierno catalán, las Icrea, o las del País Vasco, Ikerbasque. Pero aunque estas plazas no dependen de la burocracia universitaria, el salario no es competitivo" (Mujer, 42 años, EUA, 16 años en el extranjero).

\section{Discusión y conclusiones}

A partir de la experiencia biográfica de los propios protagonistas, en este trabajo hemos intentado desvelar las razones de la diáspora científica española en el campo disciplinar de la investigación sobre Comunicación. Los miembros de esta red implícita de investigadores ofrecen un punto de vista aventajado para observar desde la distancia la consideración que en el exterior tiene la investigación sobre Comunicación realizada en España. Además de un diagnóstico, también se ha solicitado a los entrevistados un pronóstico, una propuesta de soluciones para mejorar la investigación en Comunicación española. Nos hemos aproximado específicamente al fenómeno a la "fuga de talento" hacia países que ocupan una posición central en el mercado académico global. La crisis financiera sufrida en España entre 2008 y 2012 ha provocado también una importante emigración académica desde España hacia universidades latinoamericanas (México, Ecuador, Colombia o Chile), cuya dimensión e impacto está todavía por analizar.

En la percepción que los investigadores entrevistados para este trabajo tienen de las limitaciones que aquejan a la academia española, pueden distinguirse aspectos sistémicos, generalizables a otras disciplinas científicas, y aspectos idiosincráticos, propios del área de Comunicación. El problema de la endogamia y la ausencia de salarios competitivos son comunes a toda la universidad española y merecen la calificación de sistémicos. Son problemas conocidos por la opinión pública española, objeto de debate periódico en la prensa y codificados en documentos como el Informe sobre el retorno e incorporación de científicos e investigadores a España (Josa-Prado; Gómez-Sebastián, 2018). El sistema universitario español se revela autopoiético en la concepción de Luhmann (1997): solo es entendido (y defendido) por algunos de sus integrantes, normalmente aquellos que alcanzan posiciones centrales en el propio sistema. Las críticas provienen, mayoritariamente, de los outsiders; o mejor dicho, de los outcasts, los expulsados del sistema.

Lamentablemente, el sistema de acreditación implantado por la Aneca con carácter general desde 2008 no parece revelarse como una solución al nepotismo y las redes clientelares, sino que es posible que incluso esté dificultando todavía más la incorporación de investigadores extranjeros. Nuestros entrevistados señalan una paradoja ya avanzada por Afonso (2016) al comentar este tipo de mecanismos supuestamente meritocráticos de acceso y progresión en la carrera académica:

"Este sistema nacional de control de calidad, originalmente diseñado para limitar la endogamia, a menudo actúa como una barrera burocrática para los outsiders, y no previene realmente el reclutamiento endogámico local" (Afonso, 2016, p. 819).

Quizá los hallazgos más interesantes de este trabajo sean los que tienen que ver estrictamente con el propio campo de la investigación sobre Comunicación. A juicio de los entrevistados, la academia española en esta disciplina se sitúa al margen del discurso científico internacional. La idea de 'investigación de frontera', la que pretende ensanchar los límites de lo conocido, sería en gran media ajena a los comunicólogos españoles. No formaría parte de su 'habitus', en términos de Bourdieu.

Un factor limitante, a decir de nuestros informantes, es la incardinación de los estudios y la investigación sobre Comunicación en las humanidades, lo que distancia a la academia española del centro de gravedad cuantitativo que domina las ciencias sociales. Es este un debate interesante al que no escapan otros países como Francia, cuya orgullosa insularidad 
no le impide fascinar al resto de la academia global con el pensamiento original de unos Bruno Latour o Thomas Piketty. Lo que sí parece aconsejable es demandar unas cualificaciones mínimas a aquellos estudiantes de Comunicación que pretendan desempeñarse como investigadores, garantizando una mínima formación estadística en los planes de estudio de las maestrías conducentes a programas de doctorado. En un contexto en el que los propios periodistas de datos parecen demandar esa formación, los departamentos de Comunicación tendrán que abrirse a las ciencias sociales computacionales, tanto para la formación profesional de los reporteros como para el adiestramiento de futuros investigadores.

Por último, los investigadores entrevistados para este trabajo insisten en desmontar una de las excusas más habituales para explicar la falta de diálogo entre la investigación española y la del Norte Global: el dominio del inglés. Si los académicos españoles tienen dificultades para colocar sus trabajos en revistas científicas de impacto no es porque no puedan expresar adecuadamente lo que quieran decir, sino porque lo que dicen no tiene el suficiente interés o la mínima calidad requerida por los revisores. Faltaría esa ambición de conectarse con las teorías y métodos más actuales y vigentes para situar las investigaciones en los problemas por resolver, en el afán de atreverse a reconocer y ampliar la frontera del conocimiento.

Los investigadores españoles en el extranjero son prueba de que, como advertía Chambliss (1989), el talento no es un regalo celestial inexplicable, o el resultado de trabajar más que los demás. La excelencia, entendida como una consistente superioridad en la competición, es la consecuencia de hacer las cosas de manera diferente. Ubicado en instituciones nacionales (universidades u otros centros), el investigador sobre Comunicación en España rara vez parece preguntarse dónde está la frontera del conocimiento en su campo. En la medida en que el acceso al empleo permanente suele estar casi siempre constreñido a la misma institución en la que ha comenzado y seguido su trayectoria académica, la clave de la progresión laboral no se encuentra tanto en el logro de la excelencia científica cuanto en la sintonía, cuando no en la obsecuencia, con quienes pueden facilitarle una adecuada progresión en su carrera profesional. No parece, pues, haber incentivos para hacer y pensar diferente -la clave de la excelencia para Chambliss-, sino para ser una acomodaticia pieza más del statu quo.

\section{Referencias}

Academic Careers Observatory (2018). “USA, Academic career structure”. Max Weber Programme. Florencia: European University Institute.

https://www.eui.eu/ProgrammesAndFellowships/AcademicCareersObservatory/AcademicCareersbyCountry/USA

Aceituno-Aceituno, Pedro; Danvila-del-Valle, Joaquín; González-García, Abel; Bousoño-Calzón, Carlos (2018). “Entrepreneurship, intrapreneurship and scientific mobility: The Spanish case". PLoS one, v. 13, n. 9, pp. 1-20.

https://doi.org/10.1371/journal.pone.0201893

Aceituno-Aceituno, Pedro; Melchor, Lorenzo; Danvila-del-Valle, Joaquín; Bousoño-Calzón, Carlos (2017). “Cumulative advantages and social capabilities in scientific mobility in the Health Sciences: The Spanish case". PLoS one, v. 12, n. 3, e0173204.

https://doi.org/10.1371/journal.pone.0173204

Aceituno-Aceituno, Pedro; Romero-Martínez, Sonia J.; Víctor-Ponce, Patricia; García-Núñez, José (2015). “Factores determinantes de la movilidad de investigadores españoles en Ciencias de la Salud". Medicina clínica, v. 144, n. 11, pp. 520-524.

https://doi.org/10.1016/j.medcli.2014.10.020

Afonso, Alexandre (2016). "Varieties of academic labor markets in Europe". PS: Political science \& politics, v. 49, Special issue 4, pp. 816-821.

https://doi.org/10.1017/S1049096516001505

Albarrán, Pedro; Carrasco, Raquel; Ruiz-Castillo, Javier (2017). "Geographic mobility and research productivity in a selection of top world economics departments". Scientometrics, v. 111, n. 1, pp. 241-265.

https://doi.org/10.1007/s11192-017-2245-x

Álvarez, Pilar; Vázquez, Cristina (2018). “Profesores universitarios desde 300 euros”. El país, 12 febrero, pp. 18-19. https://elpais.com/politica/2018/02/09/actualidad/1518207100_741157.html

Amoedo-Souto, Carlos; Nogueira-López, Alba (2013). "Regateando hacia la excelencia: tasa de reposición de efectivos y universidades públicas". Revista española de derecho administrativo, v. 157, pp. 249-278. 
Appelt, Silvia; Van-Beuzekom, Brigitte; Galindo-Rueda, Fernando; De-Pinho, Roberto (2015). Which factors influence the international mobility of research scientists?. París: Organización para la Cooperación y el Desarrollo Económicos (OCDE).

https://doi.org/10.1787/5js1tmrr2233-en

Arenilla-Sáez, Manuel (2012). “La gobernanza del sistema universitario español”. En Manuel Arenilla Sáez (dir.). La reforma de la universidad española: un análisis desde su gobernanza, pp. 65-250. Oleiros, A Coruña: Netbiblo.

Aunión, J. A. (2013). “La movilidad penaliza al aspirante a una plaza de docente universitario". El país, 14 octubre, p. 40. https://elpais.com/sociedad/2013/10/13/actualidad/1381691428_943981.html

Banas, Monika (2018). "Reverse brain drain - a real threat or a speculative narrative? The case of the United Kingdom and the United States". Società-Mutamento-Politica. Rivista Italiana di Sociologia, v. 9, n. 18, pp. 383-399.

https://doi.org/ 10.13128/SMP-24357

Bermejo, José-Carlos (2009). “Cosmopolitismo medieval: Bolonia”. La voz de Galicia, 29 diciembre, p. 17.

Bourdieu, Pierre (2008). Homo academicus. Madrid: Siglo XXI. ISBN: 9788432313370

Caballé, Jordi (2014). “Endogamia universitaria: efectos y remedios”. El país, 1 diciembre. https://elpais.com/politica/2014/12/01/actualidad/1417424577_931721.htm/

Carrasco, Raquel; Ruiz-Castillo, Javier (2019). "Spatial mobility in elite academy institutions in economics: the case of Spain". Series - Journal of the Spanish Economic Association, v. 10, n. 2, pp. 141-172. https://doi.org/10.1007/s13209-019-0189-5

Catanzaro, Michele (2013). "Menos de uno de cada diez científicos españoles en el extranjero creen que volverán". El periódico, 10 julio.

https://www.elperiodico.com/es/ciencia/20130710/diaspora-cientificos-espanoles-recortes-2491369

Chambliss, Daniel F. (1989). "The mundanity of excellence: An ethnographic report on stratification and olympic swimmers". Sociological theory, v. 7, pp. 70-86.

https://doi.org/10.2307/202063

De-Azúa, Félix (2014). “Un partido de profesores”. El país, 1 diciembre, p. 27.

https://elpais.com/elpais/2014/11/28/opinion/1417202506_176244.html

Demeter, Marton (2019). "So far, yet so close: International career paths of communication scholars from the Global South". International journal of communication, v. 13, pp. 578-602.

https://ijoc.org/index.php/ijoc/article/view/10181

Deville, Pierre; Wang, Dashun; Sinatra, Roberta; Song, Chaoming; Blondel, Vincent D.; Barabási, Albert-László (2014). "Career on the move: Geography, stratification, and scientific impact". Scientific reports, v. 4, art. 4770. https://doi.org/10.1038/srep04770

Doquier, Frédéric; Rapoport, Hillel (2012). "Globalization, brain drain, and development". Journal of economic literature, v. 50, n. 3, pp. 681-730.

https://doi.org/10.1257/jel.50.3.681

Dustmann, Christian; Preston, Ian P. (2019). "Free movement, open borders, and the global gains from labor mobility". Annual review of economics, v. 11, pp. 783-808.

https://doi.org/10.1146/annurev-economics-080218-025843 C

Elorza-Moreno, Ana; Melchor, Lorenzo; Orts-Gil, Guillermo; Gracia, Cristina; Lacunza, Izaskun; Izquierdo, Borja; Fernández-Vera, José-Ignacio (2017). "Spanish science diplomacy: A global and collaborative bottom-up approach". Science \& diplomacy, v. 6, n. 1.

http://www.sciencediplomacy.org/article/2017/spanish-science-diplomacy-global-and-collaborative-bottom-approach

Fundación Cotec para la Innovación (2019). Informe Cotec 2019.

http://informecotec.es/media/INFORME-COTEC-2019_versionweb.pdf

García-Romero, Antonio (2012). "Influencia de la carrera investigadora en la productividad e impacto de los investigadores españoles. El papel de la ventaja acumulativa”. Revista española de documentación científica, v. 35, n. 1, pp. 38-60. https://doi.org/10.3989/redc.2012.1.762

Hasselbach, Jacob A. (2019). "Framing brain drain: between solidarity and skills in European labor mobility". Review of international political economy, v. 26, n. 6, pp. 1333-1360.

https://doi.org/10.1080/09692290.2019.1626755

Jauralde-Pou, Pablo (2008). “Universidad, decadencia”. El mundo, 5 abril, pp. 4-5. 
Josa-Prado, Fernando; Gómez-Sebastián, Silvia (coords.) (2018). Informe sobre el retorno e incorporación de científicos e investigadores a España (IRICIE). Madrid Científicos Retornados a España (CRE).

https://cre.org.es/iricie

Kuhn, Peter; McAusland, Carol (2006). The international migration of knowledge workers: When is brain drain beneficial?. Cambridge (USA): National Bureau of Economic Research. NBR Working Paper No. 12761.

https://www.nber.org/papers/w12761

López-Rejas, Javier (2016). “Eduardo Oliver: 'Con recursos, España puede consolidarse como una potencia científica'”. El cultural, 26 abril.

https://elcultural.com/Eduardo-Oliver-Con-recursos-Espana-puede-consolidarse-como-una-potencia-cientifica

Luhmann, Niklas (1997). Organización y decisión: autopoieisis, acción y entendimiento comunicativo. Barcelona: Anthropos. ISBN: 9788476585177

Marín, Maribel; Álvarez, Pilar (2014). “La endogamia alcanza al 73\% de los docentes”. El país, 1 diciembre, pp. 30-31. https://elpais.com/politica/2014/11/28/actualidad/1417196515_775495.html

Martínez-Nicolás, Manuel (2020). “La investigación sobre comunicación en España (1985-2015). Contexto institucional, comunidad académica y producción científica”. Revista latina de comunicación social, n. 75, pp. 383-414.

https://www.doi.org/10.4185/RLCS-2020-1432

Montero-Sánchez, María-Dolores (1994). La información periodística y su influencia social. Barcelona: Universitat Autònoma de Barcelona. ISBN: 8433569503

Moro-Martín, Amaya (2017). "How dare you call us diplomats". Nature, v. 543, p. 289.

https://www.nature.com/news/how-dare-you-call-us-diplomats-1.21628

Parey, Matthias; Ruhose, Jens; Waldinger, Fabian; Netz, Nicolai (2017). "The selection of high-skilled migrants". The review of economics and statistics, v. 99, n. 5, pp. 776-792.

https://doi.org/10.1162/REST_a_00687

Peñafiel-Saiz, Carmen; Ronco-López, Milagros; Videla-Rodríguez, José-Juan; Echegaray-Eizaguirre, Lázaro (2019). “Percepción y análisis de la comunidad universitaria sobre el sistema actual de investigación de la comunicación en España”. Revista latina de comunicación social, n. 74, pp. 1521-1541.

https://doi.org/10.4185/RLCS-2019-1397

PerImutter, David (2010). Promotion and tenure confidential. Cambridge (USA): Harvard University Press. ISBN: 9780 674048782

Saperas, Enric (2016). "Cuatro décadas de investigación comunicativa en España. Los procesos de institucionalización y profesionalización de la investigación”. Disertaciones. Anuario electrónico de estudios en comunicación social, v. 9, n. 2, pp. $27-45$. https://doi.org/10.12804/disertaciones.09.02.2016.02

Segarra, Pablo (2019). "España recupera los niveles de inversión en I+D previos a la crisis económica”. 20 Minutos, 23 diciembre.

https://www.20minutos.es/noticia/4093312/0/espana-recupera-los-niveles-de-inversion-en-i-d-previos-a-la-crisiseconomica

Silió, Elisa (2019). "La universidad ahuyenta el talento extranjero por sus sueldos y papeleo". El país, 7 febrero. https://elpais.com/sociedad/2019/02/06/actualidad/1549479775_916820.html

The economist (2011). "The magic of diasporas". The economist, 19 Nov.

https://www.economist.com/leaders/2011/11/19/the-magic-of-diasporas

Weiss, Robert S. (1994). Learning from strangers: The art and method of qualitative interview studies. Nueva York: Simon \& Schuster. ISBN: 9780684823126

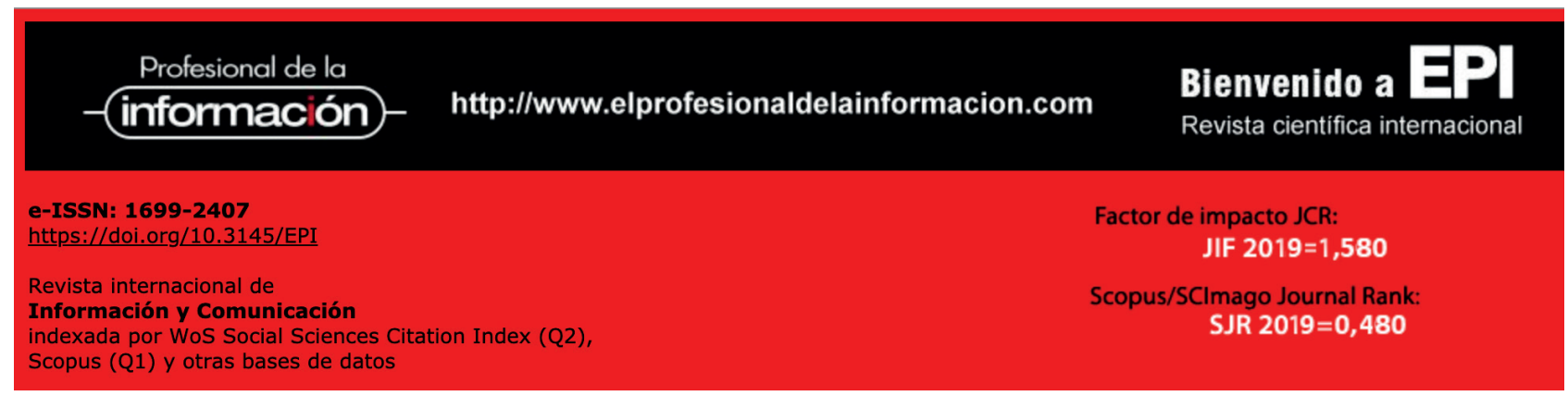

